\title{
Rehabilitation nach Kniegelenkersatz
}

\section{Dass Patienten nach der Versorgung mit einer Knieendoprothese unbe- dingt Rehabilitationsmaßnahmen brauchen, dürfte unbestritten sein. Doch wie intensiv müssen diese Maßnahmen sein? Und wie viel dür- fen sie kosten?}

- Finnische Forscher randomisierten 86 Patienten nach totaler Kniegelenksarthroplastie in zwei Gruppen. Die Experimentalgruppe wurde mit einer intensiven, multidisziplinären Rehabilitation versorgt, während die Kontrollgruppe wie üblich rein orthopädisch nachbehandelt wurde.

Nach zwölf Monaten waren beide Optionen, gemessen an der selbst berichteten funktionellen Kapazität der Patienten, etwa gleich erfolgreich. Ein deutlicher Unterschied bestand jedoch in den Kosten. In der Kontrollgruppe lagen sie um 1830 Euro pro Patient signifikant niedriger. Die Autoren meinen daher, dass eine multidisziplinäre Rehabilitation bei diesem Patientengut nicht $\mathrm{zu}$ rechtfertigen sei.

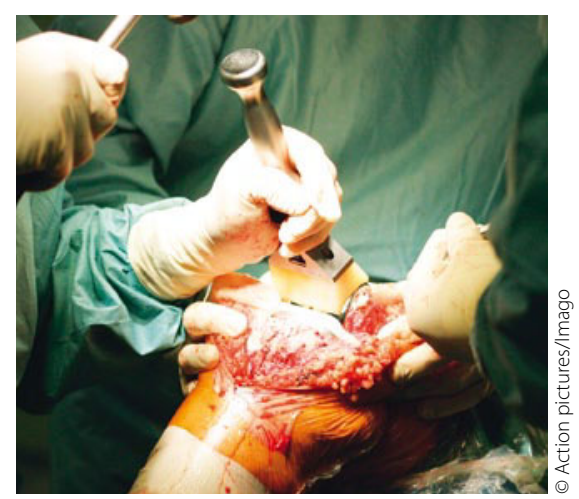

Wie viel Reha braucht dieser Patient nach seinem Kniegelenksersatz?

\section{Kommentar}

Die Fallzahl dieser Studie ist recht niedrig. Aussagen über therapeutische Äquivalenzen sind mit solch kleinen Studien nicht zu treffen. Die Frage muss erlaubt sein, ob beide Rehabilitationsprogramme gleich wirksam oder gleich unwirksam waren. Dass eine intensive, multidisziplinäre Reha mehr kostet, als das übliche Vorgehen, erstaunt wohl kaum jemanden. Wichtig wäre es zu klären, ob es Patientengruppen gibt, für die das eine Programm besser ist als das andere. Fazit: Ich glaube, dass die multidisziplinäre Reha mit diesen Daten nicht ad acta gelegt werden sollte.

E. ERNST

\section{- A. M. Kauppila et al.}

Economic evaluation of multidisciplinary rehabilitation after primary total knee arthroplasty based on a randomized controlled trial. Arthritis Care Res. (Hoboken). 63 (2011) 311-464

\section{Symptomatik des engen Spinalkanals}

\author{
Eine Auswertung von vier Studien \\ mit 741 Patienten mit Spinalkanal- \\ stenose gibt Hinweise auf die \\ typische Symptomatik dieser \\ Patienten und trägt zur rationellen \\ Diagnostik bei.
}

— Eine Literatursuche einschlägiger, zwischen Januar 1966 und September 2010 veröffentlichten Publikationen brachte vier Studien zu Tage, bei denen die Symptomatik von 741 Patienten mit lumbaler Spinalkanalstenose analysiert wurde.

Für die Diagnose eines engen Spinalkanals sprechen ein Alter über 70, tief sitzende Rückenschmerzen und Schmerzen an der Rückseite beider Oberschenkel und im Gesäß beim Stehen und Gehen, die sich beim Bücken nach vorne und beim Hinsetzen rasch bessern. Auch ein Taubheitsgefühl und eine Schwäche der Wadenmuskulatur sollten an eine Spinalkanalstenose denken lassen.

Patienten mit dieser Symptomenkonstellation sollten umgehend $\mathrm{zu}$ einer Schnittbilduntersuchung, zum Beispiel Computertomografie oder Magnetresonanztomografie überwiesen werden. Für die klinisch relevante Diagnose des engen Spinalkanals sind sowohl klinisch-neurologische Symptome als auch der Nachweis des engen Kanals in der Bildgebung erforderlich. Es kommt nämlich nicht selten vor, dass zwar in der Bildgebung eine Einengung des Spinalkanals festgestellt wird, der betroffene Patient aber keinerlei Beschwerden aufweist.

Tief sitzende Rückenschmerzen alleine reichen jedenfalls nicht aus, um an eine Stenose des Spinalkanals denken zu lassen, da bei dieser Symptomatik mus- kuläre und entzündliche Ursachen wesentlich häufiger verantwortlich sind.

\section{Kommentar}

Die Studie zeigt einmal mehr, wie wertvoll eine sorgfältige Anamnese unter Berücksichtigung von auslösenden und lindernden Faktoren für eine rationale Diagnostik ist. Leider wird die Kunst der Anamnese im Medizinstudium nicht ausreichend gelehrt und von vielen Ärzten - teils aus Unkenntnis, teils aus Zeitmangel - nicht in der gewünschten Weise gepflegt.

H. S. FüESSL . 